\title{
La importancia del marco de las coaliciones promotoras en el análisis de políticas públicas contemporáneo
}

Ruth Martinón*

Palabras clave: Análisis de políticas públicas, marco de las coaliciones promotoras, ideas, sistemas de creencias, aprendizaje orientado a las políticas.

A partir de la década de los años ochenta, el análisis politológico de políticas públicas experimentó cambios suficientemente relevantes para que hablemos del comienzo de una nueva etapa, marcada por una mayor profundidad teórica y metodológica. Son varios los autores que han protagonizado este proceso; no obstante, aquí defenderemos la importancia singular del marco de las coaliciones promotoras (en adelante, MCP), ideado por Paul A. Sabatier, con la especial colaboración de Hank C. Jenkins-Smith ${ }^{1}$, y desarrollado con trabajos de otros muchos investigadores que lo han aplicado a múltiples dominios de políticas.

Paul A. Sabatier no sólo es el principal artífice de la que consideramos la mejor aportación al análisis de políticas públicas contemporáneo. Al mismo tiempo, es el autor que se muestra más consciente del proceso que se está gestando en el seno del análisis de políticas públicas, y así lo ha hecho explícito en su propio trabajo.

\section{El marco de las coaliciones promotoras: sus premisas y objeto de estudio}

El MCP es una propuesta teórica para el estudio del cambio de las políticas públicas concebida para que pueda ser aplicable a la mayoría de las poliarquías industriales. Introduce las ideas como variable independiente dentro de un conjunto de otros factores, tanto dinámicos como estructurales, con los que se aspira a abarcar toda la complejidad del proceso de las políticas públicas. Se parte de la crítica y el intento de supera-

* Profesora del Departamento de Derecho Financiero y del Trabajo de la Universidad de La Laguna. Doctora en Ciencia Politica y de la Administración por la Universidad Carlos III de Madrid.

${ }^{1}$ La obra más representativa fue editada por ambos autores (1993). 
ción de la heurística de división en fases del proceso de políticas que ha prevalecido ampliamente en dicho análisis, al tiempo que se aspira a elaborar una teoría explicativa del complejo proceso de las políticas públicas, desde el convencimiento de que tal labor es una tarea pendiente, aunque cada vez menos alejada, de la Ciencia Política.

El MCP se dotó, desde el principio, con unas premisas, y concretó una serie de hipótesis que las sucesivas aplicaciones empíricas del modelo han ido precisando. Es necesario exponer brevemente las premisas, en tanto son concebidas como «prerrequisitos para cualquier teoría del cambio en las políticas» (Sabatier 1993: 35).

En primer lugar, se considera la necesidad de tener en cuenta el papel que juega «la información técnica respecto a la magnitud y las facetas del problema, sus causas, y los impactos probables de las diversas soluciones» (Sabatier y Jenkins-Smith 1999: 118). Refleja la importancia que se concede a las ideas, concretadas en los aspectos cognitivos y, dentro de éstos, en los técnicos.

En segundo lugar, ha de tratarse del estudio del cambio a lo largo de al menos una década, porque así lo exige la comprensión del papel que el aprendizaje tiene en el proceso del cambio en las políticas. Tampoco el corto plazo es adecuado para comprender la importancia relativa de factores tales como los cambios en las condiciones socioeconómicas, ni permite abarcar el ciclo completo de la política pública, como desea Sabatier.

En tercer lugar, se considera que el modo más útil para estudiar el cambio en las políticas sobre tal período de tiempo es a través de la consideración de los subsistemas de politicas como unidad de análisis, que además serán de naturaleza intergubernamental e incluirán como actores, no sólo a funcionarios, parlamentarios y miembros de los grupos de interés, sino también a periodistas, investigadores y analistas de políticas que jueguen papeles importantes en la generación, difusión y evaluación de las ideas de las políticas públicas.

Por último, las políticas públicas son conceptualizadas como sistemas de creencias, en la medida en que incorporan teorías implícitas sobre cómo lograr sus objetivos. Como veremos, es esto lo que permite analizar el traslado de los cambios de los sistemas de creencias de las élites y las coaliciones a las políticas. Se trata, en definitiva, de premisas requeridas por el deseo de aprehender la complejidad del cambio en las políticas públicas.

No es extraño encontrarse con estudios de políticas públicas que tratan los cambios que se dan en éstas, sin hacer explícito que el cambio en las políticas sea su objeto de estudio. Precisamente, la consideración explícita de los grandes cambios de las políticas como objeto de estudio es una característica propia de la mayoría de los trabajos de políticas contemporáneos y, especialmente, del MCP.

Para analizar el cambio, el MCP parte de la distinción entre los factores externos e internos al subsistema de la política. A su vez, dentro de dicho subsistema, encontramos las coaliciones promotoras en las que unos subconjuntos de actores defienden diferentes propuestas de políticas en ese campo. El argumento básico es que mientras el aprendizaje orientado a las políticas de los miembros de esas coaliciones es un aspecto importante del cambio en las mismas y puede, con frecuencia, alterar aspectos secundarios del sistema de creencias de la coalición, los cambios en los aspectos nucleares de una política son, generalmente, resultado de perturbaciones en factores no cognitivos externos al subsistema, tales como las condiciones macroeconómicas o el ascenso de una nueva coalición gobernante en el sistema.

El MCP no trata de indagar el origen último de las políticas (cosa, por otro lado, que, tal vez, ni tenga sentido plantear). Tampoco hace una equiparación entre cambio y cualquier producto o impacto, de modo que no hubiese distinción más que de grado entre cambio y estabilidad. La concreción del cambio en el sistema de creen- 
cias en el que se configura la política pública objeto de estudio, facilita la percepción de esa dicotomía, de modo que hay cambio si se producen transformaciones en el sistema de creencias que estamos considerando que es la política pública. Es más, se termina (Sabatier 1998) denominando cambio mayor al que afecta a los aspectos nucleares de la política, y cambio menor, al que se da en los aspectos secundarios.

El MCP es pues un marco teórico causal, que tras explicitar un conjunto de premisas, presenta una serie de variables independientes o factores explicativos, es decir, que se encuentran en la causa de la que sería la variable dependiente: el cambio en las políícas públicas, concretado del modo en que hemos visto.

La amplitud y diversidad de esas variables independientes muestran que se trata de un marco teórico en gran medida ecléctico. Es cierto que se pone el énfasis en el estudio del aprendizaje orientado a las políticas y, en general, en los elementos cognitivos, pero sus mismas conclusiones nos remiten a los aspectos políticos y socioeconómicos como variables fundamentales. Así, el cambio en la política pública dentro de un subsistema puede ser entendido como el producto de dos procesos: en primer lugar, los esfuerzos de las coaliciones promotoras que se encuentran en el subsistema, por trasladar sus sistemas de creencias a los programas de acción gubernamental, para lo cual buscan poder. El segundo proceso es una perturbación externa, es decir, los efectos de sucesos sistémicos sobre los recursos y constricciones de los actores del subsistema (Sabatier 1987: 679).

En definitiva, el cambio en las políticas es visto como reflejo de las fluctuaciones de los sistemas de creencias de las coaliciones dominantes de un subsistema de políticas dado. El aprendizaje y el análisis de políticas puede afectar a los aspectos secundarios de los sistemas de creencias, pero los cambios fundamentales en la política pública requieren, generalmente, alteraciones en los parámetros no cognitivos del sistema total. Esto es así porque para poder plasmar los sistemas de creencias en las políticas públicas, se requieren recursos y oportunidades, y éstos dependen, principalmente, de las alteraciones de los parámetros sistémicos no cognitivos.

\section{El subsistema de política como unidad de análisis}

El MCP adopta como unidad de análisis lo que denomina subsistema de una política pública, definido como el conjunto de actores que provienen de una variedad de organizaciones públicas y privadas, y que están activamente involucrados en una cuestión o problema de una política (Sabatier 1988: 131). Podemos observar su ubicación en el sistema político global en el cuadro 1, que recoge las variables y la conexión entre las mismas que esgrime el MCP.

Hay varios aspectos que sirven para precisar el concepto de subsistema que maneja el MCP y que le diferencia de otras unidades de análisis que han sido utilizadas en los estudios de los procesos de elaboración de políticas públicas. Tal vez el más relevante sea el tipo de actores que abarca: pueden ser públicos o privados, pertenecer a diversos niveles de gobierno y a una amplia variedad de actividades o profesiones. De hecho, una de las características más innovadoras del MCP es que desafía la asunción implícita de la mayoría de los politólogos de que la afiliación organizacional de un actor es primordial.

El MCP considera fundamental distinguir el subsistema de la política pública del sistema político global en el que aquél se inserta, además de diferenciar los factores estables de los dinámicos. De este modo, encontramos que, a lo largo del tiempo, el cambio en las políticas puede considerarse función de tres variables: los parámetros relativamente estables del sistema, los elementos externos al subsistema de carácter dinámico, y la propia dinámica interna del subsistema. 


\section{CUADRO 1}

\section{El marco de las coaliciones promotoras}

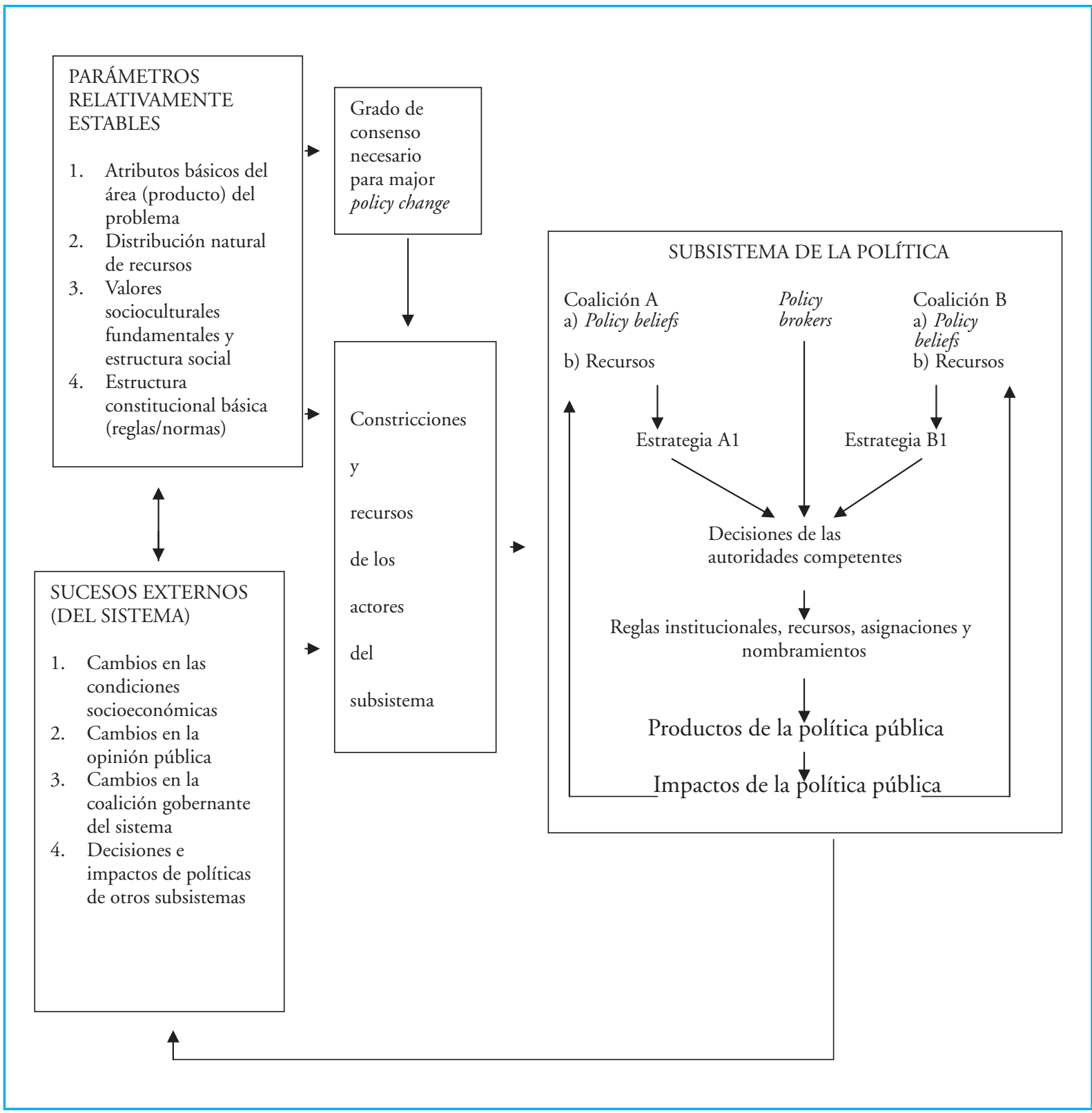

Fuente: Elaboración propia a partir de Sabatier (1999b: 145).

Los elementos relativamente estables son los aspectos estructurales en los que se desenvuelve el quehacer político, bien por constituir las reglas del juego del mismo, bien por ser parte de la realidad en la que se desarrolla. En concreto, se consideran los siguientes aspectos:

a) Atributos básicos del área del problema o del producto objeto de la política.

b) Distribución básica de los recursos naturales.

c) Valores socioculturales fundamentales y estructura social.

d) Estructura básica constitucional y reglas fundamentales. 
Se trata de factores que pueden estar tanto dentro como fuera del subsistema de la política pública que es objeto de estudio. Afectan claramente al comportamiento de los actores, en la medida en que les suponen constricciones y recursos, en el sentido de que epueden limitar la gama de alternativas posibles o, si no, afectar a los recursos y creencias de los actores del subsistema» (Sabatier 1993: 20).

El cambio de estos elementos no es imposible, pero sí muy difícil, de modo que rara vez son el objetivo de las estrategias de las coaliciones (excepto en el muy largo plazo). Precisamente, porque para cambiarlos se necesita el esfuerzo concentrado de una coalición promotora de al menos una década, lo más probable es que los cambios en la políitca pública dentro de un subsistema provengan de los factores dinámicos externos al mismo.

Los valores socioculturales fundamentales, al igual que la estructura social, son fruto del pasado y se necesitarían largos períodos de tiempo para que cambiasen. Los grupos sociales que se encuentran en una situación de desventaja, aunque luchen para cambiar su situación en el largo plazo, en sus estrategias en el corto plazo han de tener en cuenta esa circunstancia como un hecho estructural. Además, las normas socioculturales fundamentales representan aquellos sistemas de creencias que han trascendido a sus propias coaliciones promotoras y estructuran el comportamiento de sistemas políticos enteros (Munro 1993: 112). Suelen citarse las diferencias entre Europa y Estados Unidos en cuanto al papel que se considera posible que asuma el Estado, en el entendido de que en los países europeos se dan grados de intervención, por ejemplo, sobre los medios de producción, difíciles de imaginar en el país norteamericano.

La inclusión de la estructura constitucional básica nos sitúa en el esquema de los diferentes niveles de Ostrom (1990: 50), de modo que nos encontraríamos en el macro nivel centrado en las estructuras constitucionales, que afecta al modo en que se configuran las decisiones colectivas. Se trata de reglas que también pueden determinar la extensión del aprendizaje orientado a las políticas; en este sentido, nos interesa destacar cómo los sistemas descentralizados coadyuvan al aprendizaje al facilitar la comparación de las distintas experiencias llevadas a cabo por gobiernos subnacionales distintos.

Schlager (1995: 260) critica que los autores del MCP no hacen explícitas las conexiones entre estos parámetros estables del sistema y la formación y las actividades de las coaliciones promotoras, excepto en cuanto afectan al aprendizaje, y sugiere la posibilidad de introducir la teoría de la elección estructural de Moe (1990), que estudia los efectos de los parámetros estables de un sistema (por ejemplo, diferenciando un sistema presidencialista de uno parlamentario) sobre las estrategias de una coalición promotora para alcanzar sus objetivos.

En definitiva, se trata de factores que se asumen. Implican la asunción del aspecto estructural del escenario donde cobran vida las políticas públicas, y nos muestran que, al igual que la escuela de la elección racional institucional, acepta que las reglas institucionales afectan al comportamiento individual.

Los elementos dinámicos externos al subsistema en el MCP constituyen los prerrequisitos críticos para que se dé el cambio mayor en las políticas públicas. Son los siguientes:

a) Cambios en las condiciones socioeconómicas y tecnológicas.

b) Cambios en la opinión pública.

c) Cambios en la coalición gobernante en el sistema.

d) Impactos y decisiones de las políticas de otros subsistemas.

Se trata de cambios que pueden ser realmente importantes en el curso de algunos pocos años o una década. Alteran las constricciones y las oportunidades de los actores confrontados en el subsistema y representan un conti- 
nuo desafío a dichos actores, que deben aprender a anticiparse a ellos y afrontarlos de acuerdo a sus creencias básicas y sus intereses.

Los trabajos empíricos muestran que los sucesos exógenos no alteran por sí mismos, directamente y sin ambigüedad, los recursos y oportunidades de los actores del subsistema. Sino, más bien, «tales sucesos son interpretados por los actores del subsistema y, después, explotados con mayor o menor habilidad» (Sabatier y JenkinsSmith 1993: 221).

Se considera que las décadas de investigación en políticas públicas, sugieren que el público en general juega un papel más modesto en la formulación e implantación de las políticas que lo que sugieren las prioridades de investigación de la disciplina. Aunque haya una correlación bastante fuerte entre los cambios importantes en la opinión pública y los cambios en la dirección general de la política gubernamental y afecte a la dirección general de la política pública, el margen de maniobra sigue siendo inmenso (Sabatier y Jenkins-Smith 1999: 148).

Los cambios en la coalición gobernante de determinado nivel de gobierno se refieren a los cambios electorales importantes, puesto que se considera que requiere que la misma coalición controle el poder ejecutivo y las cámaras parlamentarias. Los impactos y decisiones de las políticas de otros subsistemas hay que tenerlos en cuenta porque ninguno de éstos es autónomo. De hecho, las decisiones e impactos de otros sectores de políticas son los elementos dinámicos que más afectan a los subsistemas específicos.

Se trata, en definitiva, de factores que al poder cambiar sustancialmente en períodos no muy largos de tiempo, se constituyen en la causa principal de cambios en los recursos, oportunidades y constreñimientos de los actores de un subsistema, y, por tanto, necesariamente, están detrás de los grandes cambios en las políticas públicas.

\section{La dinámica interna del subsistema: coaliciones promotoras y sistemas de creencias}

Para adentrarnos en la dinámica interna del subsistema debemos comenzar con el concepto de coalición promotora, el cual se aleja del concepto de coalición política (sea gubernamental, parlamentaria o electoral) que implica un pacto entre partidos políticos (Reniu 2002: 60), y se acerca a los conceptos de grupo y de red, como reflejo de la interrelación entre actores públicos y privados que se genera en la arena de las políticas públicas. De hecho, la literatura sobre las ideas en las políticas públicas con frecuencia ha resaltado el papel de este tipo de coaliciones, por la importancia que los grupos sociales suelen tener en el éxito o fracaso político de las ideas nuevas (Calvin y Velasco 1997: 172).

Las coaliciones promotoras son definidas como «los actores de una amplia variedad de instituciones que comparten las creencias del núcleo de la política y que coordinan su comportamiento de diversas maneras» (Sabatier y Jenkins-Smith 1999: 130). Podríamos decir que son un actor político colectivo, en la medida en que reunirían las características de asociación voluntaria, con estabilidad relativa de su actividad, comunidad de intereses y objetivos, y una línea de acción coordinada. Sin embargo, el hecho de que carezcan de organización como tales y, por tanto, no tengan medios identificables para la toma de decisiones globales sobre las que se les puedan exigir responsabilidades, cuestiona su consideración como actor (Sibeon 1999: 141). De este modo, aparte de su cercanía al concepto de red o de grupo en sentido amplio (como en el caso del concepto de subsistema), observamos su clara diferenciación con los grupos de interés (actores colectivos con organización estable). Sin embargo, su configuración reticular, estructuración difusa y dinamismo nos recuerda más a un movimiento social, especialmente a los denomina- 
dos movimientos monotemáticos, vinculados a la resolución de un problema específico. Si bien se aleja del concepto de movimiento social caracterizado como opositor a las élites y la autoridad², y la preferencia por la utilización de recursos no convencionales en su actuación.

Sin embargo, no todos los actores que podemos encontrar en un subsistema de política pertenecen a una de las coaliciones promotoras del mismo. Hay algunos investigadores que, indiferentes a las disputas de la política pública, pueden participar simplemente porque tienen ciertas habilidades que ofrecer; $\mathrm{o}$ burócratas que realmente se adhieren a la tradición de la competencia neutral. Además, encontramos a los «intermediarios de la política», 0 policy brokers, cuya principal preocupación es mantener el nivel del conflicto político dentro de límites razonables y poder alcanzar alguna solución sensata al problema. La distinción entre promotor e intermediario, sin embargo, descansa en un continuo (Sabatier 1993: 27).

Hay que tener en cuenta que las sucesivas investigaciones empíricas confirman que igual que los científicos no son necesariamente 'neutrales' o 'indiferentes respecto a la política pública' (y, por ello, frecuentemente son miembros de una coalición política), las agencias administrativas generalmente defienden posiciones más moderadas que los grupos de interés que son sus aliados.

El número de coaliciones promotoras que podemos encontrar en un subsistema, varía. «En los subsistemas quietos, inactivos, latentes, puede haber una única coalición. En la mayoría de los casos, sin embargo, habrá de dos a cuatro coaliciones importantes» (Sabatier 1993: 26).

Respecto al papel que juegan o cómo actúan las coaliciones promotoras, se considera que esos actores que comparten un conjunto de creencias básicas buscan realizarlas, es decir, trasladarlas a los programas públicos, influyendo en el comportamiento de las instituciones gubernamentales a lo largo del tiempo. Para ello, las coaliciones actúan en una multiplicidad de escenarios ${ }^{3}$ (parlamentos, gobiernos, agencias administrativas y tribunales, de diferentes niveles de gobierno). Cuentan con diversos instrumentos: la alteración de las reglas y presupuestos de las agencias, el cambio del papel que juegan los personajes clave, el cambio en la opinión pública (a través de los medio de comunicación) o los intentos de alterar comportamientos a través de manifestaciones o boicots, por ejemplo trabajando a través del mercado en lugar de directamente sobre el gobierno; o, el intento de alterar gradualmente las percepciones de una diversidad de actores a través de la investigación y el intercambio de información (Sabatier y Jenkins-Smith 1999: 142).

Por otro lado, cuando las coaliciones intentan trasladar sus creencias a las políticas públicas, las presiones para alcanzar un compromiso suelen llevar a programas gubernamentales que incorporan elementos de diferentes coaliciones promotoras. De hecho, aunque, normalmente habrá una coalición dominante y una o más coaliciones minoritarias, en cualquier sistema intergubernamental, coaliciones diferentes pueden tener el control de diversas unidades de gobierno.

El aspecto del MCP que ha sido más criticado es cómo se forman y mantienen las coaliciones a lo largo del tiempo. En un principio, el MCP asumió implícitamente que los actores que compartían creencias similares del núcleo de la polííca, actuarían de modo coordinado; de manera que los sistemas de creencias eran el aglutinador principal de las coaliciones.

${ }^{2}$ Los movimientos sociales plantean «una acción directa disruptiva contra las élites, las autoridades u otros grupos o códigos culturales. Aunque lo más habitual es que esta disrupción sea pública, también puede adopta la forma de resistencia personal coordinada o de reafirmación colectiva de nuevos valores» (Tarrow 1997: 22).

${ }^{3}$ Los autores de las coaliciones promotoras adoptan la expresión «venue shopping» acuñada por Baumgartner y Jones (1993). 
Schlager (1995) y Schlager y Blomquist (1996) realizaron las principales críticas en este sentido a través del material propio de la literatura de la acción colectiva y sugirieron tener en cuenta otros factores que afectan a los costes de interacción: cuanto menores fuesen éstos, más fácil sería la coordinación. Sabatier (1998) asumió en parte estas consideraciones, pero también las rebatió. En primer lugar, se estableció la diferencia entre cooperación fuerte y débil (en esta última los costes de interacción se verían fácilmente compensados por la confianza mutua que genera el compartir el núcleo de la política) (Zafonte y Sabatier 1998). En segundo lugar, se recordó que el modelo de individuo del marco de las coaliciones promotoras permite aumentar los beneficios percibidos y reducir los costes de coordinación, a través del devil shift (o truco del diablo): la tendencia de los actores en las situaciones de mucho conflicto a percibir a sus oponentes como más perversos y más poderosos de lo que probablemente son (Sabatier et al. 1987).

Dentro de los subsistemas de políticas, un concepto clave para el MCP son los sistemas de creencias, concebidos como conjuntos de prioridades de valor y asunciones causales, teorías implícitas sobre cómo alcanzar los objetivos. Son las estructuras de pensamiento y opinión de las élites de las políticas públicas. «Incluyen prioridades de valor, percepciones de la importancia de las relaciones causales, percepciones sobre el estado del mundo (incluyendo la magnitud del problema), percepciones sobre la eficacia de los instrumentos, etcétera» (Sabatier 1993: 17).

El concepto de sistema de creencias concreta la introducción de las ideas en este análisis de las políticas públicas. Asimismo, en la medida en que las políticas públicas o programas incorporan teorías implícitas sobre como alcanzar sus objetivos (Pressman y Wildawsky 19984; Majone 1980), también ellos pueden ser concebidos como sistemas de creencias. Se trata de una de las premisas del MCP y viene a significar que el concepto de sistema de creencias se usa, «como la plantilla sobre la cual se mide el cambio, tanto respecto a las creencias de las diferentes coaliciones como el contenido real de la política pública» (Jenkins-Smith y Sabatier 1993a: 55). La posibilidad de colocar creencias y políticas sobre la misma plantilla, suministra una herramienta para apreciar la influencia de varios actores a lo largo del tiempo, y, particularmente, el papel de las ideas sobre el cambio en las políticas.

La relevancia teórica y metodológica que los sistemas de creencias tienen para el MCP, en tanto los cambios en su estructura anunciarán los intentos de su proyección en la política pública, dicha estructura se establece cuidadosamente. Se asume que en los sistemas de creencias se puede distinguir un núcleo de unos elementos secundarios. En concreto, el sistema de creencias está organizado en una estructura tripartita y jerárquica (Sabatier 1998: 103) como se aprecia en el Cuadro 2.

El núcleo profundo del sistema de creencias compartidas incluye creencias normativas y ontológicas básicas, tales como la valoración relativa de la libertad individual versus la igualdad social, que opera a lo largo de, virtualmente, todos los dominios de políticas. La familiar escala izquierda/derecha opera a este nivel.

A continuación encontramos las creencias del núcleo de la politica, que representan compromisos normativos y percepciones causales básicos de la coalición a lo largo de un subsistema o dominio de política completo. Incluyen prioridades de valor fundamentales; percepciones básicas concernientes a la seriedad general del problema y sus principales causas; y estrategias para realizar valores nucleares dentro del subsistema. Estas creencias del núcleo de la política son el aglutinador fundamental de las coaliciones porque representan los compromisos empíricos básicos en el dominio de especialización de las élites de la política pública.

${ }^{4}$ «Las políticas implican teorías. Ya sea que se declaren explícitamente o no, las políticas apuntan hacia una cadena de causas que aparecen entre las condiciones esenciales y las consecuencias futuras» (Pressman y Wildawsky 1998: 56). 


\section{CUADRO 2}

Estructura revisada de los sistemas de creencias de las élites de las politicas públicas

\begin{tabular}{|c|c|c|c|}
\hline $\begin{array}{l}\text { Características definitorias } \\
\text { Extensión } \\
\text { Susceptibilidad al cambio } \\
\text { Componentes ilustrativos }\end{array}$ & $\begin{array}{l}\text { Núcleo profundo } \\
\text { Axiomas normativos } \\
\text { fundamentales y ontológicos } \\
\text { A lo largo de todos los subsistemas } \\
\text { de políticas } \\
\text { Muy difíili; semejante a una } \\
\text { conversión religiosa } \\
\text { 1. La naturaleza humana: } \\
\text { a) Inherentemente malo vs. } \\
\text { Socialmente redimible } \\
\text { b) Parte de la naturaleza vs. } \\
\text { Dominio sobre la } \\
\text { naturaleza } \\
\text { c) Egoístas intolerantes vs. } \\
\text { Contractualistas } \\
\text { 2. Prioridad relativa de varios } \\
\text { valores últimos: libertad, } \\
\text { seguridad, poder, } \\
\text { conocimiento, salud, amor, } \\
\text { belleza, etc. } \\
\text { 3. Criterios básicos de } \\
\text { distribución de la justicia: ele } \\
\text { bienestar de quién es } \\
\text { importante? El peso relativo de } \\
\text { uno mismo, el grupo primario, } \\
\text { todo el mundo, generaciones } \\
\text { futuras, otras especies, etc. } \\
\text { 4. Identidad sociocultural (por } \\
\text { ejemplo, de etnia, religión, } \\
\text { género, profesión) }\end{array}$ & $\begin{array}{l}\text { Núcleo de la política } \\
\text { Posiciones en la política } \\
\text { fundamentales, concernientes a las } \\
\text { estrategias básicas para la } \\
\text { consecución de valores nucleares } \\
\text { dentro del subsistema } \\
\text { En todo el subsistema } \\
\text { Difícil, pero puede ocurrir si la } \\
\text { experiencia revela serias anomalías } \\
\text { Preceptos normativos } \\
\text { fundamentales: } \\
\text { 1. Orientación sobre las } \\
\text { prioridades de valor básicas } \\
\text { 2. Identificación de grupos u } \\
\text { otras entidades cuyo bienestar } \\
\text { es de la máxima preocupación } \\
\text { Preceptos con un componente } \\
\text { empírico substancial: } \\
\text { 3. Sobre todo, la seriedad del } \\
\text { problema } \\
\text { 4. Causas básicas del problema } \\
\text { 5. Distribución adecuada de } \\
\text { autoridad entre gobierno y } \\
\text { mercado } \\
\text { 6. Distribución adecuada de } \\
\text { autoridad entre los niveles de } \\
\text { gobierno } \\
\text { 7. Prioridad respecto a los } \\
\text { diversos instrumentos de } \\
\text { políticas públicas (p. ej., } \\
\text { regulación, seguros, } \\
\text { educación, pagos directos, } \\
\text { créditos fiscales) } \\
\text { 8. Método de financiación } \\
\text { 9. Capacidad de la sociedad para } \\
\text { resolver el problema (p. ej., } \\
\text { competición de suma cero vs. } \\
\text { potencial para la mutua } \\
\text { adaptación; pluralismo } \\
\text { tecnológico vs. cargos electos) } \\
\text { 10. Participación del público } v s . \\
\text { expertos vs. cargos electos } \\
\text { 11. Policy core policy beliefs }\end{array}$ & $\begin{array}{l}\text { Aspectos secundarios } \\
\text { Decisiones instrumentales e } \\
\text { investigaciones informativas } \\
\text { necesarias para la implantación del } \\
\text { núcleo de la políitica } \\
\text { Generalmente, sólo en parte del } \\
\text { subsistema } \\
\text { Moderadamente fácil; este es el } \\
\text { tema de la mayor parte del policy- } \\
\text { making administrativo e, incluso, } \\
\text { legislativo } \\
\text { 1. Seriedad de aspectos específicos } \\
\text { del problema en espacios } \\
\text { específicos } \\
\text { 2. Importancia de los diversos } \\
\text { nexos causales en diferentes } \\
\text { espacios y a lo largo del tiempo } \\
\text { 3. La mayoría de las decisiones } \\
\text { referidas a las reglas } \\
\text { administrativas, disposiciones } \\
\text { presupuestarias, disposición de } \\
\text { casos, interpretación } \\
\text { estatutaria, e, incluso, revisión } \\
\text { estatutaria } \\
\text { 4. Información referida a la } \\
\text { puesta en marcha de programas } \\
\text { específicos o instituciones }\end{array}$ \\
\hline
\end{tabular}

Fuente: Elaboración propia a partir de Sabatier (1999b: 113).

Por último, los aspectos secundarios del sistema de creencias de la coalición comprenden un largo conjunto de creencias que no abarcan todo el subsistema, concernientes a la seriedad del problema, la importancia relativa de diversos factores causales en escenarios específicos, preferencias de políticas en cuanto a regulaciones deseables 0 repartos presupuestarios, el diseño de instituciones específicas, las evaluaciones... 
No hay que olvidar que, a diferencia del uso que hacen de la expresión «sistema de creencias» otros autores, en el MCP se trata de los sistemas de creencias de las élites. Los subsistemas están compuestos por las élites de las políticas más que por los miembros del público en general, de modo que se puede considerar que la mayor parte de los actores tiene sistemas de creencias relativamente complejos e internamente consistentes respecto al área de la política pública de su interés (Sabatier 1993: 30).

\section{El modelo de individuo y el aprendizaje orientado a las políticas}

El modelo de individuo del MCP, fundamental para explicar su construcción teórica, ha sido en gran medida modificado a partir de las críticas y aportaciones, principalmente de Schlager, pero también de otros autores, y de la misma evidencia empírica. La consecuencia principal es el mayor desarrollo de un modelo de individuo y un tratamiento más detenido de la relación entre intereses y creencias.

Desde un principio se asumió que los actores son instrumentalmente racionales, esto es, que buscan usar información y otros recursos para alcanzar sus fines; aunque simpatizan mucho más con la investigación en psicología cognitiva y social que con los trabajos económicos. Schlager y Blomquist (1996: 660) lo relacionan con la racionalidad procedimental de Simon (1985). «En particular, el MCP asume que los fines son generalmente complejos y que la capacidad de un individuo para percibir el mundo y procesar la información se ve afectada por sesgos y constricciones cognitivos» (Sabatier y Jenkins Smith 1999: 130).

Respecto a la relación entre creencias e intereses, aunque se ha avanzado en concreción se han mantenido las ideas básicas: los actores no se guían primordialmente por simples fines del propio interés económico o político, y las preferencias del interés propio no son fáciles de averiguar. Por el contrario, normalmente, los fines del actor son complejos y deben averiguarse empíricamente (Sabatier 1998: 109).

Otro concepto clave para entender el funcionamiento interno del subsistema de políticas es el aprendizaje orientado a las politicas. De hecho, tal vez, el aspecto más ambicioso del MCP es la integración de la literatura sobre la utilización del conocimiento y la literatura sobre el cambio en las políticas públicas. En el fondo, o, al menos, en el origen, se trata de un marco conceptual para demarcar el papel que juega el análisis de políticas en el aprendizaje orientado a las políitcas y el papel que juega tal aprendizaje, por tanto, en el cambio de las políticas a lo largo de periodos de una década o más.

Siguiendo a Heclo (1974: 306), el MCP considera que el aprendizaje orientado a las políticas es el conjunto de alteraciones relativamente resistentes de pensamiento o de intenciones de comportamiento, que son resultado de la experiencia, y que están relacionados con la obtención y revisión de preceptos del sistema de creencias de individuos o colectividades (tales como las coaliciones promotoras) y, por tanto, de los objetivos de la política.

Sin embargo, «A causa de la resistencia al cambio de las creencias centrales, [...], tal aprendizaje orientado a las politicas queda normalmente confinado a los aspectos secundarios de los sistemas de creencias» (Jenkins-Smith 1993a: 5). Cuando los actores se encuentran con constricciones u oportunidades, intentan responder de modo coherente con su núcleo de política. Además, el filtro perceptivo, que es un componente fundamental del modelo de individuo del $\mathrm{MCP}$, hace que los actores se opongan a la información que sugiere «que sus creencias del núcleo profundo o del núcleo de la política, pueden ser nulas y/o inasequibles, y, normalmente, usarán análisis de políticas formal para apoyar y elaborar esas creencias (o atacar a los puntos de vista de sus oponentes)» (Sabatier 1998: 104). 
En definitiva, aunque los sucesos exógenos o las actividades de los oponentes pueden, eventualmente, forzar el examen de creencias centrales, la mayor parte del aprendizaje se manifiesta en los aspectos secundarios de un sistema de creencias o de un programa gubernamental.

El MCP asume que el aprendizaje orientado a las políticas es instrumental, esto es, que los miembros de diversas coaliciones buscan entender mejor el mundo y los efectos de las diversas intervenciones gubernamentales de cara a promover sus objetivos en la política pública.

Todo este proceso de aprendizaje orientado a las políticas se produce en el escenario del proceso político, donde las personas compiten en la asignación de valores a través de los instrumentos del poder político (caracterizado por contar, aunque sea como último recurso, con el uso de la fuerza) en su beneficio. Por tanto, «este proceso no es una búsqueda desinteresada de la verdad» (Jenkins-Smith y Sabatier 1993a: 45).

La idea fundamental es que en los sistemas con poder disperso es difícil desarrollar una posición mayoritaria a través del puro ejercicio del poder. Por ello, suele ser necesario convencer a los otros actores de la bondad de nuestra posición y las consecuencias de las otras alternativas. Esto no sólo de aborda con el proceso de argumentación descrito por Majone (1989), sino, especialmente, a través de la utilización de la información, el análisis, la investigación, y, por tanto, el aprendizaje.

Para Sabatier (1987: 671), sólo los individuos pueden aprender; cuando se habla del aprendizaje de las coaliciones se es consciente de la utilización de una metáfora. A su vez, al tratar el proceso de aprendizaje se distingue entre el aprendizaje dentro de una misma coalición promotora y, por tanto, un mismo sistema de creencias, que se muestra como relativamente poco problemático; y, el aprendizaje entre coaliciones.

Se entiende que los cambios en los sistemas de creencias de una coalición, generalmente, comenzarán con el aprendizaje de los individuos; encontrarán, primero, la resistencia de las dinámicas de grupo; y, después, serán difundidas a través del mismo. La difusión dependerá de la cantidad de movimientos de personal, la compatibilidad de la información con las creencias existentes, lo persuasiva que sea la evidencia y las presiones políticas que haya para el cambio.

El aprendizaje entre los sistemas de creencias de distintas coaliciones se produce en su interrelación. El estudio de dicho proceso de aprendizaje intenta identificar las condiciones bajo las cuales es probable que se produzca un debate analítico productivo entre miembros de diferentes coaliciones promotoras. El indicador de que tal tipo de debate se ha producido es el hecho de que ambas coaliciones lleguen a alterar aspectos centrales de sus sistemas de creencias, o, al menos, aspectos secundarios muy importantes, como resultado del diálogo más que por un cambio en las condiciones externas.

Según los autores, la probabilidad de tal aprendizaje es función de al menos tres variables (Jenkins-Smith y Sabatier 1993a: 48): Primero, el nivel de conflicto (que refleja el grado de incompatibilidad de las creencias centrales de las coaliciones que compiten); segundo, la medida en que el tema se pueda tratar de modo analítico; $y$, tercero, la naturaleza del foro de análisis, según si es más abierto o más profesional. El mayor grado de aprendizaje se dará con bajo nivel de conflicto, facilidad analítica del problema y profesionalidad del foro. 


\section{El MCP y otros enfoques contemporáneos de políticas públicas}

El MCP que acabamos de presentar puede encuadrarse en lo que Jordan (1990b) ha denominado los enfoques de subsistemas de políticas, desarrollados en las décadas de los setenta y ochenta alrededor de conceptos como redes, teoría de juegos, policy communities, policy networks... No obstante, al mismo tiempo forma parte de ese conjunto de trabajos que muestran el incipiente desarrollo de teorías causales explicativas del proceso de las políticas y, que se centran en la explicación de los grandes cambios en las políticas públicas, prestando especial interés al papel de las ideas. De hecho, «la literatura sobre la importancia de las ideas en los cambios y procesos de formación de las políticas ha crecido [...] en el fértil terreno de los análisis existentes sobre actores y sus interacciones» (Subirats y Gomà 1998: 25).

Veamos ahora cuáles son las notas distintivas de estas investigaciones y propuestas teóricas de políticas públicas que nos hacen considerar que se abre una nueva etapa. Hay que destacar, además del propio MCP de Sabatier y Jenkins-Smith, los trabajos de la escuela de la elección racional institucional, el enfoque de las corrientes múltiples de Kingdon y la teoría del equilibrio interrumpido de Baumgartner y Jones (véase Cuadro 3).

\section{CUADRO 3}

Las nuevas propuestas de análisis de políticas públicas

\begin{tabular}{|l|l|l|l|l|}
\hline Modelo & $\begin{array}{l}\text { Marco de las coaliciones } \\
\text { promotoras }\end{array}$ & $\begin{array}{l}\text { Elección racional } \\
\text { institucional }\end{array}$ & $\begin{array}{l}\text { Enfoque de las corrientes } \\
\text { múltiples }\end{array}$ & $\begin{array}{l}\text { Teoría del equilibrio } \\
\text { interrumpido }\end{array}$ \\
\hline Autor representativo & Sabatier & Ostrom & Kingdon & Baumgartner y Jones \\
\hline Fortalezas & $\begin{array}{l}\text { Consideración de las ideas } \\
\text { Facilita aplicación } \\
\text { empírica }\end{array}$ & $\begin{array}{l}\text { Globalidad } \\
\text { Facilita aplicación } \\
\text { empírica }\end{array}$ & $\begin{array}{l}\text { Tratamiento de las ideas en } \\
\text { la formulación de políticas }\end{array}$ & $\begin{array}{l}\text { Concepto de policy image } \\
\text { La explicación del cambio } \\
\text { radical }\end{array}$ \\
\hline Debilidades & $\begin{array}{l}\text { Explicación de la cohesión } \\
\text { de las coaliciones }\end{array}$ & Homo economicus estricto & $\begin{array}{l}\text { No facilita la aplicación } \\
\text { empírica }\end{array}$ & $\begin{array}{l}\text { No contempla el cambio } \\
\text { incremental }\end{array}$ \\
\hline
\end{tabular}

Fuente: Elaboración propia.

Para Sabatier, el modelo más relevante de todos ellos es el enfoque de la elección racional institucional, y, más concretamente, los trabajos de Ostrom ${ }^{5}$. «Su enfoque de desarrollo y análisis institucional es, probablemente, lo más cercano a una 'teoría global' que tenemos en las ciencias sociales» (Sabatier 1999b: 264). Además, considera que al igual que su MCP, Ostrom y sus compañeros han facilitado el desarrollo a largo plazo de su propuesta, a través de la elaboración teórica y la contrastación empírica abierta a la comunidad académica.

Sintéticamente, la propuesta es un análisis que pretende poder predecir el comportamiento probable de los individuos dentro de determinada estructura. Para ello se tienen en cuenta la arena de acción en la que se encuentran los individuos, en determinadas posiciones y con determinados recursos; los aspectos estructurales que condicionan dicha arena de acción; y, la información y valoración que realizan los actores, que guiarán su conducta en función de un análisis coste-beneficio a modo de la teoría económica neoclásica y la teoría de juegos.

${ }^{5}$ Lo que al mismo tiempo no está reñido con su oposición a una concepción radical de la escuela de la elección racional, lo que ha permitido la consideración del modelo de las coaliciones promotoras como una pasarela de conexión entre los estudios de políticas públicas americanos y europeos (Bergeron, Surel y Valluy 1998: 197). 
La diferencia principal entre el MCP y la elección racional institucional radica en el concepto de homo economicus que Ostrom prefiere utilizar en su análisis (Ostrom 1999: 44). Aunque Sabatier acabó modificando su teoría del individuo a partir de la comparación crítica que hacen Schlager y Blomquist (1996) de su modelo con el de Ostrom, seguirá manteniendo diferencias importantes al complejizar el homo economicus guiado por el interés, con la consideración de la importancia de sus creencias y los vínculos que establece con otras personas a partir de ellas.

Respecto al modelo de las múltiples corrientes de Kingdon, se trata de una traslación exitosa de la idea del can garbage model (Cohen et al. 1972) al ámbito de la formulación de las políticas. Entiende que éste es un proceso compuesto de tres corrientes de actores y procesos: una corriente de problemas, compuesta por los datos de varios problemas y varias propuestas de definición de problemas; una corriente de políticas públicas que incluye las propuestas de solución a los problemas de políticas; y una corriente política consistente en los procesos electorales y los cargos electos. Estas corrientes, que, generalmente, son independientes, se encuentran cuando se abre una «ventana de oportunidad» que permite que los entrepreuners de la política intenten conectar las diversas corrientes y, si tienen éxito, produzcan un cambio de política pública importante.

El aprecio de Sabatier por el trabajo de Kingdon es indudable, como uno de los pocos politólogos que ha tratado seriamente el papel de las ideas y el análisis en la elaboración de políticas públicas. Sin embargo, el MCP ve la corriente 'analítica' mucho más integrada con la corriente 'política', que lo que las ve Kingdon; trata el proceso de las políticas públicas en su totalidad, no sólo la elaboración de la agenda y la formulación de la política; y trata de relacionar las 'ventanas de oportunidad' de Kingdon para el cambio mayor con tipos específicos de cambios en los elementos exógenos al subsistema de la política (Sabatier 1993: 37).

Con todo, la crítica más dura que Sabatier realiza a Kingdon es que, a diferencia de él y de Ostrom, no ha facilitado lo que considera un aspecto fundamental, probablemente, el más importante, para el desarrollo de una teoría a largo plazo. «:Por qué el enfoque de las corrientes múltiples de Kingdon (inicialmente publicado en 1984) atrajo a mucha menos contrastación empírica y sufrió mucha menos reelaboración?» (Sabatier 1999b: 266) ${ }^{6}$. Principalmente, porque no ha perseguido conscientemente una estrategia que anime a otros colegas a contrastar sus propuestas para ir modificando la concepción inicial, lo que se muestra en la misma forma de exposición del enfoque. No están claras o bien especificadas cuáles son las variables dependiente o independiente, no se identifica bien qué actores podemos encontrar en cada corriente, y tampoco está claro el modelo de individuo.

Respecto a la Teoría del Equilibrio Interrumpido de Baumgartner y Jones (1993), por un lado, se da una coincidencia en la reivindicación del papel de las ideas; por otro lado, es un modelo que también se centra expresamente en algunas fases en tanto enfatiza dos elementos relacionados del proceso de políticas: la definición de problemas y la elaboración de la agenda.

Los autores argumentan que el proceso de elaboración de políticas públicas en Estados Unidos, se caracteriza por largos periodos de cambio incremental que son interrumpidos por breves periodos de grandes cambios de políticas. Esto sucede cuando quienes están interesados en el cambio saben aprovechar la difusión de nuevas policy images a través de los múltiples escenarios de políiticas característicos de los Estados Unidos.

En este modelo las ideas son fundamentales para las elites de cara al mantenimiento del orden establecido alrededor de un tema. Se utiliza el concepto de policy image para referirse a cómo es entendida una política pública. Nos recuerdan a los sistemas de creencias al ser descritos como «una mezcla entre información empírica y atraccio-

${ }^{6}$ De hecho, aunque Kingdon es citado con mucha frecuencia, Sabatier sólo conoce que N. Zaharadis «haya aplicado realmente y de forma crítica el enfoque de las múltiples corrientes de una manera seria» (Sabatier 1997: 7). 
nes emotivas» (Baumgartner y Jones 1993: 26), de modo que diferentes personas tiene diferentes imágenes de la misma política. Lo importante es que la creación y mantenimiento de un monopolio de política está íntimamente ligado a la creación y mantenimiento de una imagen de política de apoyo. Lo cual nos recuerda también al paralelismo que el MCP hace entre la estructura de los sistemas de creencias y las de las políticas públicas. De hecho, éste modelo también es expresamente deudor de Heclo y de Majone.

Sin embargo, existe una diferencia muy importante entre el MCP y la teoría del equilibrio interrumpido: en el MCP los sistemas de creencias y las preferencias cambian gradualmente a lo largo del tiempo en la medida en que los individuos son persuadidos a aceptar los argumentos de otros, o acumulan información a través de la experiencia, de modo que se genera un cambio incremental; mientras en la teoría del equilibrio interrumpido, el cambio en la elección individual y, consecuentemente, en la política pública, puede ser rápido y radical (Schlager 1999: 244).

\section{El nuevo análisis de políticas públicas}

Estos nuevos enfoques tienen en común el que asumen la finalidad de una teoría politológica del proceso de políticas públicas, lo que sería «explicar cómo los actores políticos interesados interactúan dentro de las instituciones políticas para producir, implementar, evaluar y revisar las políticas públicas» (Schlager y Blomquist 1996: 653).

Afirma Sabatier que el cambio fundamental es de actitud. Se trata de asumir, primero, que «algo tan complejo como el proceso de políticas públicas requiere lentes simplificadoras que nos digan qué buscar y qué ignorar; y, en segundo lugar, que esas lentes deberían ser explícitas más que implícitas» (Sabatier 1990b: 271).

Además, aunque, generalmente, se centran en algunos aspectos del proceso de las políticas públicas (muchas veces, implícitamente), se trata de modelos que ambicionan explicar el proceso de las políticas públicas en general, o que, al menos, no constriñan su construcción conceptual a la idea de las fases, y de este modo representan una superación de tal heurística. Incluso en el caso del modelo de Kingdon, centrado claramente en la formación de la política (elaboración de la agenda y toma de decisiones) es concebible extenderlo a la totalidad del proceso (Zaharadis 1990).

Al mismo tiempo y, correlativamente, son modelos que logran reunir los criterios mínimos que se le exigen a una teoría científica, son sometidos a contraste empírico, no evitan ninguno de los principales elementos que los politólogos consideran importantes en el proceso de elaboración de políticas (por eso manejan múltiples variables explicativas) y, aunque puedan existir elementos normativos, son, fundamentalmente, teorías positivas que buscan explicar lo mejor posible el proceso de políticas públicas.

Por último, aunque tal vez sea lo más llamativo de esta generación de estudios de políticas públicas, encontramos la irrupción que significa la consideración de las ideas como variable explicativa. Ante la pregunta de por qué cambian las políticas, se hacen con un espacio muy importante de la respuesta, las ideas, los valores, la argumentación, los aspectos normativos...

Por supuesto, la Ciencia Política se ha ocupado de estos aspectos al estudiar las ideologías, los valores, la cultura política... tanto de los miembros de la clase política como de las sociedades en general. En los años sesenta el concepto de cultura política de Almond y Verba (1963) permitía introducir en el ámbito de los estudios empíricos el mundo de los valores, de las ideas, de las percepciones políticas (Botella 1997); y Converse (1964) introdujo 
el análisis sistemático de los sistemas de creencias políticos. Sin embargo, en el análisis de política públicas supone una irrupción su consideración como variable explicativa del cambio. John (1998: 144) lo explica así:

«Lo que es interesante para el teórico de las políticas públicas no es que las ideas existan, sino si juegan un papel independiente en el proceso de las políticas. [...] Las aproximaciones de políticas públicas basadas en las ideas, arguyen que son las ideas que estos actores traen a la esfera política, la razón del cambio de la política o de su estabilidad. La razón por la cual hay determinadas políticas es porque la gente cree e intenta influir en los decisores políticos sobre la base de que hay un curso de acción acertado. Esta promoción de las ideas propias es un factor causal sobre los efectos de la política de las instituciones e intereses políticos».

En este aspecto, como en otros (por ejemplo, el tratamiento de los grandes cambios como objeto de estudio, o la amplitud de los actores considerados, o incluso, en la crítica a la heurística de las fases) los trabajos de Hugh Heclo son un precedente fundamental. Introduce el papel de las ideas, el conocimiento y el aprendizaje en la explicación del cambio en las políticas en su explicación de las políticas sociales de Gran Bretaña y Suecia (Heclo 1974), y con su concepto de issue network se introduce la confrontación de los elementos cognitivos frente al interés:

«Cualquier interés material directo es con frecuencia secundario al compromiso intelectual y emocional. Los miembros de la red refuerzan mutuamente su opinión acerca de los problemas de acuerdo con sus propios intereses, más que los intereses definan sus posturas sobre los problemas (como defenderían los modelos políiticos o económicos estándares) (Heclo 1978: 102).

Otra aportación destacada en la introducción de las ideas en los estudios de las políticas públicas es la de Majone (1989: 213) quien considera que los cambios en las creencias y los valores pueden jugar un papel tan importante en el desarrollo de las políícas como los intereses económicos y políticos. «Una buena teoría del desarrollo de políticas requiere que se preste atención a las ideas, a las teorías u argumentos, al igual que a la tecnología, la economía y la política».

Tampoco podemos olvidar la conocida teoría de las comunidades epistémicas de Haas (1992) o el concepto de paradigma de la acción pública o de las políticas públicas. Es especialmente significativo el trabajo que realizó Hall (1989 y 1993), inspirado en el conocido trabajo de sociología de la ciencia de Kuhn (1962), para investigar los cambios de la política económica de varios países, considerando como un factor explicativo fundamental las teorías económicas al uso, sin tampoco negar la existencia de conflictos en razón de intereses materiales? ${ }^{7}$ De hecho, la mayor parte de estos autores que han buscado entender el aspecto normativo de las políticas públicas, lo han hecho poniendo en conexión las ideas o los valores y los intereses (Considine 2005: 52).

La publicación del libro Public Policy and Political Ideas, editado por Braun y Busch (1999), es una muestra significativa del peso que las ideas han cobrado en los modelos teóricos explicativos de las políticas públicas, al lado de las instituciones y los intereses. La amplitud de miras con las que presenta el tema, nos sirve, entre otras cosas, para visualizar la pluralidad de nuevas visiones que aporta la perspectiva de las ideas.

Las ideas son instrumentos de persuasión y legitimación de la acción política; pero también son los marcos cognitivos que estructuran y guían la acción políica. Las ideas sirven para explicar el cambio y la estabilidad de las políticas de manera complementaria a los enfoques centrados en las relaciones de poder. No hay que olvidar tampoco el puente de conexión que la consideración de las ideas en las políticas públicas establece con la literatura del

\footnotetext{
7 Véase Munro (1993) y Küebler (2000) para la comparación entre el MCP y Kuhn a través de Hall.
} 
aprendizaje (Bennett y Howlet 1992); el mismo MCP es un ejemplo destacado de ello. Se trata, además, de una nueva perspectiva que puede aplicarse a todos los campos o áreas de políticas públicas, aunque ciertamente las políticas económicas y de bienestar hayan marcado el principio de la senda, con los trabajos de Heclo y de Hall.

Por último, esta nueva atención a las ideas no sólo se ha manifestado en la faceta analítica del estudio de las políticas públicas. Fischer y Forester (1993), en The Argumentative Turn in Policy Analysis and Planning, presentan la visión práctica o aplicada de esta tendencia, al tiempo que son conscientes de las implicaciones teóricas e incluso epistemológicas y filosóficas que tiene este tema y que pueden sintetizarse con la frase con la que comienza la presentación de la obra: ¿Qué ocurre si nuestro lenguaje no es simple espejo del mundo, sino que, además y sobre todo, modela profundamente nuestra visión del mismo?

Hay que tener en cuenta cómo el estudio del papel de las ideas ha estado ligado, al menos en parte, al surgimiento de un nuevo enfoque en la Ciencia Política que busca proporcionar un modelo superior a los del antiguo institucionalismo, el pluralismo y el marxismo, considerando al Estado como un actor autónomo (Evans et al. 1985) pero que interrelaciona, cada vez más, con otros actores, en la configuración y puesta en práctica de la política pública. De ahí, el posterior desarrollo del concepto y los estudios sobre gobernanza.

\section{Conclusiones: las aportaciones del marco de las coaliciones promotoras}

El MCP fue concebido para estudiar el papel que las ideas y el aprendizaje tenían en el proceso de las políticas públicas. Para ello se elaboró un instrumental metodológico detallado muy útil para su estudio (JenkinsSmith y Sabatier 1993b), principalmente, a través del concepto de sistemas de creencias y la concepción de la política pública como concreción del mismo.

Junto a esta fortaleza metodológica, el MCP se muestra como una propuesta especialmente atractiva por varias razones. En primer lugar, porque aunque significa una defensa del papel que las ideas y el aprendizaje juegan en el cambio de las políticas públicas, abarca al resto de variables que pueden jugar un papel en dicho cambio, evitando sesgos apriorísticos. Se aspira a abarcar toda la complejidad del proceso de las políticas públicas; en ese sentido, trata de atender a todas las fases o dimensiones de la política pública (Subirats y Gomà 1998: 389), y tiene presentes todos los ámbitos en juego: el político, el simbólico y el organizativo social (Ibarra et al. 2002: 16 y 21).

En segundo lugar, porque realiza una concreción certera del concepto de cambio en las políticas y, al visualizar éstas cómo sistemas de creencias, permite apreciar el cambio que los actores logran imprimir en las mismas. El cambio se convierte en objeto explícito de estudio y es concebido de modo que se facilita su operativización.

En tercer lugar, el MCP ocupa un lugar notablemente destacado entre los estudios de políticas públicas que se vienen desarrollando desde mediados de los años ochenta por su esfuerzo de construcción teórica y la amplia aplicación empírica que ha desencadenado. Así, ha ayudado a impulsar unos estudios de políticas públicas ricos desde el punto de vista teórico y rigurosos desde el punto de vista metodológico. La propia configuración del MCP invita a su contrastación empírica, lo que ha supuesto su aplicación a múltiples dominios de política. Esas aplicaciones del modelo han permitido su progresivo perfeccionamiento.

Por último, el MCP aporta una concepción de agregación de actores, las coaliciones promotoras, que resulta más verosímil en las sociedades occidentales contemporáneas que otras concepciones anteriores, en la medida en 
que permite abarcar la multiplicidad de actores, públicos y privados, y la diversidad de relaciones entre los mismos que se generan en los escenarios de las políticas públicas. Aunque, al mismo tiempo, es probable que la mayor debilidad del MCP sea la explicación de la coordinación de los miembros de dichas agrupaciones.

\section{REFERENCIAS BIBLIOGRÁFICAS}

Almond, G. A. y Verba, S. (1963), The Civic Culture. Political Attitudes and Democracy in Five Nations. Princeton: Princeton Unviersity Press.

Baumgartner, F. R. y Jones, B. D. (1993), Agendas and inestability in American politics. Chicago y Londres: The University of Chicago Press.

Bennett, C. J. y Howlett, M. (1992), «The lessons of learning: Reconciling Theories of policy learning and policy change», Policy Sciences 25: 275-294.

Bergeron, H.; Surel, Y. y Valluy, J. (1998), «L'Advocacy Coalition Framework. Une contribution au renouvellement des études de politiques publiques?», Politix 41: 195-223.

Botella, J. (1997), «En torno al concepto de cultura política: dificultades y recursos», en P. del Castillo e I. Crespo, eds., Cultura política. Enfoques teóricos y análisis empirico. Valencia: Tirant lo Blanch.

Braun D. y Busch, A., eds. (1999), Public Policy and Political Ideas. Cheltenham y Northampton: Edward Elgar.

Calvin, C. y Velasco, J. (1997), «Las ideas y el proceso de conformación de las políticas públicas: una revisión de la literatura», Politica y Gobierno 4 (1): 169-188.

Cohen, M. March J. G. y Olsen, J. P. (1972), «A Garbage Can Theory of Organizational Choice», Adminstrative Science Quarterly 17: 1-25.

Considine, M. (2005), Making Public Policy. Cambridge: Polity Press.

Converse P. E. (1964), «The Nature of Belief Systems in Mass Publics», en D. E. Apter, ed., Ideology and Discontent. Nueva York: Free Press.

Evans, P. Ruerchemeyer, D. y Skocpol, Th., comps. (1985), Bringing The State Back In. Cambridge: Cambridge University Press.

Fischer F. y Forester J., eds., (1993), The Argumentative Turn in Policy Analysis and Planning. Durham y Londres: Duke University Press.

Gomà, R. y Subirats, J., coords., (1998), Politicas públicas en España. Contenidos, redes de actores y niveles de gobierno. Barcelona: Ariel.

Haas P. M. (1992), «Introducción: epistemic communities and internactional policy co-ordination», International Organisation 49 (1): 1-35.

Hall, P. A. (1993), «Policy Paradigms, Social Learning and the State. The case of economic policy making in Britain», Comparative Politics 25: 275-296. 
Hall, P. A., ed., (1989), The Political Power of Economic Ideas: Keynesianism across Nations. Princeton: Princeton University Press.

Heclo, H. (1974), Modern Social Politics in Britain and Sweden. From Relief to Income Maintenance. New Haven/Londres. Yale University Press.

Heclo, H. (1978), «Issue Networks and the Executive Establishment», en A. King, ed., The new American political system. Washington D. C.: American Enterprise Institute for Public Policy Research.

Ibarra, P. Goma, R. González, R. y Martí, S. (2002), «Movimientos sociales, políticas públicas y democracia radical: algunas cuestiones introductorias», en P. Ibarra, S. Martí y R. Gomà, coords., Creadores de democracia radical. Movimientos sociales y redes de politicas públicas. Barcelona: Icaria.

Jenkins-Smith, H. C. y Sabatier, P.A. (1993a), «The Dynamics of Policy-Oriented Learning», en P. A. Sabatier y H. C. Jenkins-Smith, eds., Policy Change and Learning: An advocacy coalition approach. Boulder: Westview Press.

Jenkins-Smith, H. C. y Sabatier, P. A. (1993b), «Methodological Appendix: Measuring Longitudinal Change in Elite Beliefs Using Content Analysis of Public Documents», en P. A. Sabatier y H. C. Jenkins-Smith, eds., Policy Change and Learning: An advocacy coalition approach. Boulder, Colorado: Westview Press.

John, P. (1998), «The uses and abuses of evolutionary theory in political science: a reply to Allan McConell and Keith Dowding», British Journal of Politics and International Relations 2 (1): 89-94.

Jordan, A. G. (1990b), «Policy Community Realism versus 'New' Institutionalism ambiguity», Political Studies 28: 470-484.

Kingdon, J. W. (1984), Agendas, alterantives, and public policies. Nueva York: Longman.

Küebler, D. (2000), Politique de la drogue dans les villes suisses entre ordre e santé. Analyse de conflits de mise en oevre. París y Montreal: L'Harmattan.

Kuhn, T. S. (1962), La estructura de las revoluciones cientificas. México, D. F.: Fondo de Cultura Económica.

Majone, G. (1989), Evidencia, argumentación y persuasión en la formulación de politicas. México, D. F.: Colegio Nacional de Ciencias Políticas y Administración Pública, A. C. y Fondo de Cultura Económica.

Majone, G. (1980), «Policies as theories», Omega 8: 151-162.

Moe, T. (1990), «The politics of structural choice: toward a theory of public bureaucracy», en O. Williamson, ed., Organization Theory: From Chester Barnard to the Present and Beyond. Nueva York: Oxford University Press.

Munro, J. F. (1993), «California Water Politics: Explaining Policy Change in a Cognitively Polarized Subsystem», en P. A. Sabatier y H. C. Jenkins-Smith, eds., Policy Change and Learning: An advocacy coalition approach. Boulder: Westview Press.

Ostrom, E. (1999), «Institutional Rational Choice. An assessment of the Institutional Analysis and Development Framework», en P. A. Sabatier, ed., Theories of Policy Process. Boulder: Westview Press.

Ostrom, E. (1990), Governing the Commons: The Evolution of Insititutions for Collective Action. Nueva York: Cambridge University Press. 
Pressman, J. L. y Wildawsky, A. (1998), Implementación. Cómo grandes expectativas concebidas en Washington se frustran en Oakland. México, D. F.: Colegio Nacional de Ciencias Políticas y Administración Pública, A. C. y Fondo de Cultura Económica.

Reniu Vilamala, J. M. (2002), La formación de gobiernos minoritarios en España, 1977-1996. Madrid: Centro de Investigaciones Sociológicas y Siglo XXI.

Sabatier, P. A. (1999a), ed., Theories of Policy Process. Boulder: Westview Press.

Sabatier, P. A. (1999b), «Fostering the Development of Policy Theory», en P. A. Sabatier, ed., Theories of Policy Process. Boulder: Westview Press.

Sabatier, P. A. (1998), «The advocacy coalition framework: revisions and relevance for Europe», Journal of European Public Policy 5, 1: 93-130.

Sabatier, P. A. (1997), «The Status and Development of Policy Theory: A Reply to Hill», en Policy Currents 7: 1-10.

Sabatier, P. A. (1988), «An advocacy coalition framework of policy change and the role of policy-oriented learning», en Policy Sciences 21: 129-168.

Sabatier, P.A. (1987), «Knowledge, Policy-Oriented Learning, and PolicyChange», en Knowledge: Creation, Difusión,Utilization 8 (4): 649-692.

Sabatier, P. A., Hunter, S. y Mclaughlin, S. (1987), «The Devil Shift: Perceptions and Misperceptions of Opponents», Western Political Quarterly 41: 449-476.

Sabatier, P. A. y Jenkins-Smith, H. C. (1999), «The Advocacy Coalition Framework: An Assessment», en P. A. Sabatier, ed., Theories of Policy Process. Boulder: Westview Press.

Sabatier, P. A. y Jenkins-Smith, H. C. (1993), Policy Change and Learning: An advocacy coalition approach. Boulder: Westview Press.

Schlager, E. (1999), «A Comparison of Frameworks, Theories, and Models of Policy Processes», en P. A. Sabatier, ed., Theories of Policy Process. Boulder: Westview Press.

Schlager, E. (1995), «Policy making and collective action: defining coalitions within the advocacy coalition framework», en Policy Sciences 28: 242-270.

Schlager, E. y Blomquist, W. (1996), "A comparison of three emerging theories of the policy process», Political Research Quarterly 49 (3): 651-672.

Sibeon, R. (1999), «Agency, Structure, and Social Change as Cross-Disciplinary Concepts», Politics 19 (3): 139-144.

Simon, H. A. (1985), «Human Nature in Politics: the dialogue of Psychology with Political Science», American Political Science Review 79: 293-304.

Subirats, J. y Gomà, R. (1998), «Políticas públicas: hacia la renovación del instrumental de análisis», en R. Gomà y J. Subirats, coords., Políticas públicas en España. Barcelona: Ariel.

Tarrow, S. (1997), El poder en movimiento. Los movimientos sociales, la acción colectiva y la politica. Madrid: Alianza. 
Zafonte, M. y. Sabatier, P. A (1998), «Shared Beliefs and Functional Interdependence as Determinants of Ally Networks in Overlapping subsystems», Journal of Theoretical Politics 10 (4): 473-505.

Zahariadis, N. (1999), «Ambiguity, Time, and Multiple Streams», en P. A. Sabatier, ed., Theories of Policy Process. Boulder: Westview Press. 